\title{
Foix-Chavany-Marie Syndrome as a Manifestation of Unilateral Opercular Stroke
}

\author{
$\underline{\text { Roberto Toledo-Trevino }}{ }^{1}$, Diana Manrique-Otero ${ }^{1}$, Enrique Castellanos-Pedroza ${ }^{1}$, Vanessa Cano-Nigenda ${ }^{1}$, \\ Alonso Alvarado-Bolaños ${ }^{1}$, Juan Calleja-Castillo², Antonio Arauz ${ }^{1}$ \\ ${ }^{1}$ Stroke Clinic, National Institute of Neurology and Neurosurgery Manuel Velasco Suárez, Mexico City, Mexico \\ ${ }^{2}$ Emergency Department, National Institute of Neurology and Neurosurgery Manuel Velasco Suárez, Mexico City, Mexico
}

\section{Doi: 10.12890/2021_002462- European Journal of Case Reports in Internal Medicine - ๑ EFIM 2021}

Received: 08/03/2021

Accepted: $15 / 03 / 2021$

Published: 06/04/2021

How to cite this article: Toledo-Trevino R, Manrique-Otero D, Castellanos-Pedroza E, Cano-Nigenda V, Alvarado-Bolaños A, Calleja-Castillo J, Arauz A. Foix-Chavany-Marie syndrome as a manifestation of unilateral opercular stroke. EJCRIM 2021;8: doi:10.12890/2021_002462.

Conflicts of Interests: The Authors declare that there are no competing interests.

Acknowledgements: We thank our patient for allowing us to report his case.

This article is licensed under a Commons Attribution Non-Commercial 4.0 License

\section{ABSTRACT}

Introduction: Foix-Chavany-Marie syndrome (FCMS) is a type of pseudobulbar palsy that affects facio-pharyngo-glosso-masticatory muscles. Materials and Methods: A 62-year-old man was admitted to the emergency department after 9 hours of acute dysarthria and dysphagia. MRI showed restricted diffusion in the right operculum on diffusion-weighted imaging (DWI). No thrombolytic therapy was given. The patient had a history of mechanical aortic valve replacement under anticoagulation with a vitamin K antagonist. Work-up demonstrated suboptimal levels of INR. Due to severe dysphagia during hospitalization, a percutaneous endoscopic gastrostomy (PEG) was performed.

Results: The patient was discharged 5 days later, with a modified Rankin scale (mRs) score of 3, and secondary stroke prevention. He had achieved an excellent functional outcome (mRs 1) at 6-month follow-up.

Conclusion: Our patient had a satisfactory recovery due to prompt diagnosis, secondary stroke prevention, and compliance with treatment.

\section{LEARNING POINTS}

- In the presence of acute dysarthria and dysphagia, Foix-Chavany-Marie syndrome (FCMS) should be considered.

- FCMS may occur in the presence of unilateral opercular stroke.

- Swallowing and speech therapy play an essential role in rehabilitation after the acute setting.

\section{KEYWORDS}

Foix-Chavany-Marie, pseudobulbar palsy, opercular syndrome, opercular stroke

\section{INTRODUCTION}

Foix-Chavany-Marie syndrome (FCMS) or opercular syndrome is an uncommon type of pseudobulbar palsy where bilateral damage to the operculum generates facio-pharyngo-glosso-masticatory paralysis with automatic-voluntary dissociation as a distinctive feature.

There are three main types of pseudobulbar palsy according to the site of the lesion: cortical, basal ganglia/capsular, and brainstem. FCMS corresponds to the cortical type and unlike the others, rarely presents with emotional incontinence, urinary dysfunction, or muscle tone abnormalities ${ }^{[1]}$.

It is important to consider this syndrome due to cerebrovascular disease as a possible aetiology in patients with acute onset of bilateral facial-pharyngo-glosso-masticatory paralysis. Here, we present the case of a patient with unilateral opercular stroke and FCMS. 


\section{CASE DESCRIPTION}

A right-handed 62-year-old man was admitted to the emergency department 9 hours after the acute onset of dysarthria and dysphagia. His medical history included diabetes, hypertension, chronic kidney disease, and aortic stenosis with a mechanic prosthetic valve replacement, currently under anticoagulation with a vitamin $\mathrm{K}$ antagonist. Neurological examination at admission revealed severe dysarthria with bilateral facio-pharyngo-glosso-masticatory paralysis with automatic-voluntary dissociation. The NIHSS score was 9.

\section{METHODS AND PROCEDURES}

MRI showed an area of restricted diffusion in the right operculum on diffusion-weighted imaging (DWI), suggestive of an acute ischaemic stroke. T2 FLAIR showed previous silent bilateral parietal infarcts with evidence of small-vessel disease. The brainstem had no lesions and there was no evidence of large-vessel occlusion. No thrombolytic treatment was given as the patient was outside the reperfusion therapeutic window. INR at admission was below therapeutic goals (<2). The rest of the work-up was normal: no arrhythmia was found on Holter monitoring or electrocardiogram, there were no intracavitary thrombi on echocardiogram, and carotid Doppler ultrasound was unremarkable. We concluded that cardioembolic infarction in a patient under suboptimal anticoagulation was the most feasible aetiology. As a result of severe dysphagia during hospitalization, a PEG was performed. The patient had a favourable evolution and was discharged 5 days later under oral anticoagulation and ambulatory speech and swallowing therapy. He had a modified Rankin scale (mRs) score of 3. Six months later, the patient exhibited mild dysarthria, right central facial palsy, no need for a gastrostomy tube, and excellent functional outcome with an mRS of 1 .

\section{DISCUSSION}

FCMS was described for the first time by Magnus in 1837 and detailed by Foix et al. in 1926. It is the result of bilateral cortical damage to the anterior operculum, which contains motor neurons for the cranial nerves V, VII, IX, X and XII [2]. There are few reports of unilateral damage to the anterior operculum generating FCMS, and it is believed that the presence of asynchronous contralateral corticonuclear tract lesions is the substrate of this unusual presentation ${ }^{[3]}$. There are also reports where opercular and contralateral damage to the corona radiata or brainstem causes FCMS ${ }^{[1]}$.

FCMS presents as a pseudobulbar palsy with 'autonomic voluntary dissociation' where involuntary reflex motor movements, like crying, are preserved ${ }^{[4]}$. The neuroanatomical basis of this phenomenon is explained by the presence of alternative autonomic pathways between the amygdala and motor cranial nerve nuclei interneurons located in the pontine lateral tegmentum ${ }^{[5]}$. In our case, the patient presented with an acute ischaemic stroke in the right operculum evidenced on DWI (Fig. 1) and previous left subcortical and periventricular lesions seen on T2 FLAIR (Fig. 2), which explains the existence of contralateral damage to corticonuclear tracts and thereby the presence of FCMS. In the presence of acute bilateral facial-pharyngo-glosso-masticatory paralysis, stroke should be considered as the most feasible aetiology and FCMS syndrome as a possible underlying neuroanatomical explanation. Cerebrovascular disease is the most common cause of FCMS, but there are reports with central nervous system inflammation, trauma, neurodegenerative disorders, epileptic activity, or vascular malformations as the aetiology ${ }^{[4]}$.

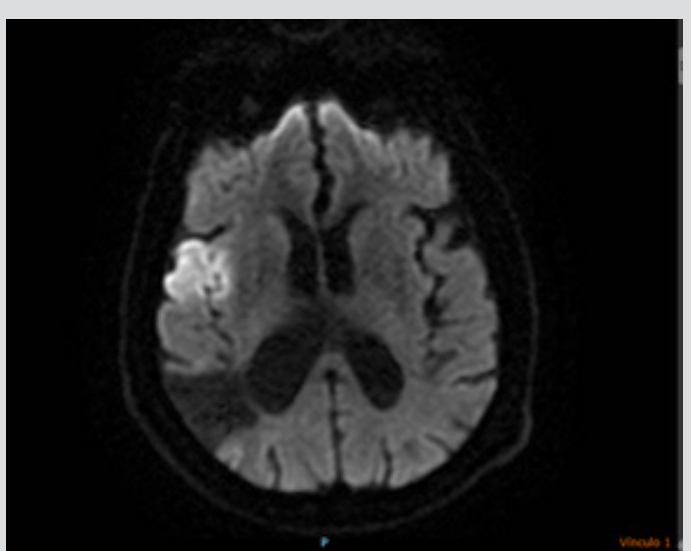

Figure 1. MRI with restriction in the diffusion-weighted imaging (DWI) sequence showing an acute right opercular infarction, as well as a previous infarction in the posterior parietal region

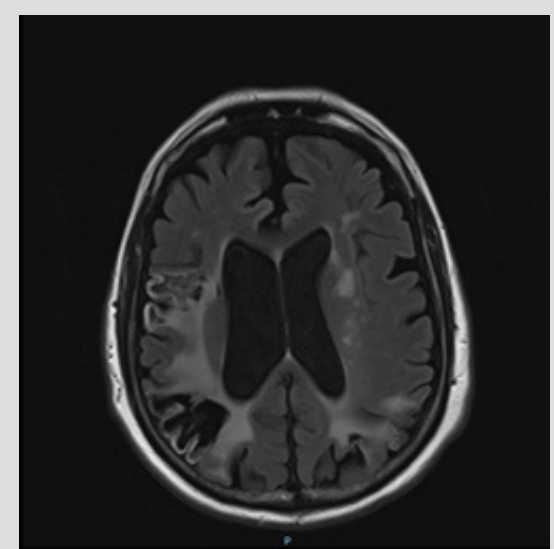

Figure 2. MRI shows positive diffuse bilateral lesions in the periventricular and subcortical white matter on the T2 FLAIR sequence 
The treatment and prognosis of FCMS are related to the aetiology. Usually, patients experience poor recovery. Airway protection and gastrostomy are cornerstones in acute settings, as patients have a significantly higher risk of developing aspiration pneumonia. Swallowing and speech therapy play an essential role in rehabilitation. Treatment of the underlying cause is the main therapeutic action, such as secondary stroke prevention ${ }^{[5]}$. Even though our patient had several comorbidities, he experienced good recovery and prognosis mainly due to prompt diagnosis and excellent treatment compliance.

\section{CONCLUSION}

The neuroanatomical substrate of FCMS is still unclear, but identifying the affected region by imaging studies can help determine the probable underlying aetiology, with treatment of the condition being the main course of action after the acute setting. Prompt identification of this syndrome and quick protection of the airway plays an important role in prognosis. Our patient achieved an excellent recovery due to secondary stroke prevention and compliance with speech and swallowing therapy.

\section{REFERENCES}

1. Yoshii F, Sugiyama H, Kodama K, Irino T. Foix-Chavany-Marie syndrome due to unilateral anterior opercular damage with contralateral infarction of corona radiata. Case Rep Neurol 2019;11(3):319-324. doi: 10.1159/000503856

2. Foix C, Chavany JA, Marie J. Diplegie facio-linguomasticatrice d'origine souscorticale sans paralysie des members. Rev Neurol 1926;33:214-219.

3. Turgut AÇ, Tubbs RS, Turgut M. French neurologists Charles Foix and Jean Alfred Émile Chavany and French pediatrician Julien Marie and the Foix-Chavany-Marie syndrome. Childs Nerv Syst 2019;36(11):2597-2598. doi: 10.1007/s00381-019-04290-1

4. Qiu X, Zhang B, Chen C, Gao T, Sun C, Xie B, Bai Y. Recovery of Foix-Chavany-Marie syndrome with multi-modal rehabilitation therapy: a case report and review of literature. Int J Phys Med Rehabil 2019,7:1 doi: 10.4172/2329-9096.1000510

5. Ohtomo R, Iwata A, Tsuji S. Unilateral opercular infarction presenting with Foix-Chavany-Marie syndrome. J Stroke Cerebrovas Dis 2012;23(1):179-181. doi: 10.1016/j. jstrokecerebrovasdis.2012.08.015 